National Board, groups had been organised for the exchange of experience, covering the metal, textile, and foodstuffs industries. The importance of securing the full co-operation of employees had clearly been demonstrated, and the close and intensive co-operation secured between the groups of chiefs and of subordinates was an essential method of organising exchange of experience. Confidence and mutual interest were the only sound basis for management research, and particularly in the Austrian method of critical visits to selected departments combined with advance or preliminary reports which were afterwards elaborated. No objections had been raised to the publication of the findings of sub-groups of experts based on these reports, and particular attention had been paid to budgetary control.

\title{
The Lister Institute.
}

\section{$\mathrm{T}$} HE Report of the governing body of the Lister Institute for 1931 refers to the various changes which have occurred in the membership of the governing body, council, and members and staff of the Institute during the past year, and describes in brief the numerous researches in which the staff and their co-workers have been engaged. A bibliography is appended to the Report, to which those interested in particular subjects may be referred for the fuller information to be obtained from original papers. Among the changes in staff may be mentioned the retirement of Sir Charles Martin and Prof. A. Harden. Prof. J. C. G. Ledingham took up the directorship of the Institute and Dr. R. Robison succeeded Prof. Harden as head of the Biochemical Department on Jan. 1.

The researches described may be roughly classified into investigations in experimental pathology and bacteriology and studies on nutritional subjects. The National Collection of Type Cultures is housed at the Institute, although the salaries of the necessary staff are furnished by the Medical Research Council : during the year, more than 5000 strains of bacteria and fungi have been distributed to workers in various parts of the world, and 200 types, including many new species, have been added to the collection. A third and enlarged edition of the catalogue of the collection is in course of preparation.

Prof. Ledingham has found in his studies on fowlpox that the Bollinger bodies seen in the epithelial cells of the lesion consist of agglomerations of Borrel bodies $(0 \cdot 25 \mu$ diameter $)$ in a lipoprotein matrix. The Borrel bodies pass through Berkefeld V filters and a single one can infect, so that it is possible that these minute bodies are the actual etiological agents. Dr. G. H. Eagles and Dr. D. MeClean have been able to cultivate vaccinia virus in a cell-free kidney extract and carry through a series of subcultures. The virus survives for considerable periods under anaerobic conditions in the cold, but filtration of the centrifuged kidney extract removes some property essential to prolonged survival. The virus can be filtered through the Berkefeld V : as with fowlpox virus, it is probable that the elementary bodies found in the lesions are the actual infecting agents.

Dr. E. W. Hurst has confirmed the observation that the virus of poliomyelitis (infantile paralysis) is transmitted along the axis-cylinders of the nerves. Monkeys can be infected with regularity by combining intrasciatic inoculation of the virus with mild trauma to the nerve. Virus and lesions first appeared in the lumbar cord, the latter first on the side of the inoculated nerve : the leg area of the opposite motor cortex was the next site to harbour virus, other areas only being involved much later. The lumbar cord is connected chiefly with the opposite motor cortex : there are only few connexions with other parts of the central nervous system. With Dr. R. W. Fairbrother, Dr. Hurst has also shown that infection with vaccinia virus does not modify a simultaneous infection with poliomyelitis. Dr. Morgan has been able to immunise two out of four horses to poliomyelitis and obtain a potent anti-serum. The potency is precipitated with the euglobulin fraction of the serum protein by adding up to 34 per cent by volume of saturated ammonium sulphate solution : the precipitate can be dialysed and redissolved in normal saline, giving a concentrated preparation which should be of great advantage in the treatment of the disease by the intraspinal route. Contacts with cases of infantile paralysis have been found to possess antibodies in their serum, although they have not developed the disease.

Work has also been carried out on the preparation of gas gangrene antitoxin, on the preparation of a dysentery toxin (Shiga) for use as a test toxin, and on the preparation of toxic extracts of the meningococcus for the purpose of titrating anti-meningococcal sera. A concentrated anti-staphylococcus serum has also been produced by the immunisation of a horse with staphylococcal filtrates.

Some preliminary work has been carried out under the auspices of the Accessory Food Factors Committee on vitamin standards. Miss Hume and Miss H. Smith have been investigating the suitability of pure recrystallised carotene as a standard for vitamin $\mathbf{A}$; Dr. H. Chick and Miss H. M. Jackson, a concentrate adsorbed on kaolin from an extract of rice polishings, provided by Dr. Jansen, as a standard for vitamin B ; and Dr. Zilva, decitrated lemon juice as a standard for vitamin C. Miss Hume, Miss Pickersgill, and Miss M. Gaffikin have been examining the standard solution of irradiated ergosterol issued by the Medical Research Council for use in the estimation of vitamin D. They find that it is stable when kept at $0^{\circ} \mathrm{C}$. Data have also been collected on the accuracy of the biological test for vitamin D. Consistent results were obtained with ten animals on each dose, good results with six, but inconsistent results with only three or four on each dose. An attempt is being made to construct a curve relating dosage and degree of calcification.

Dr. H. Schütze and Dr. S. S. Zilva have established the existence of a very definitely lowered resistance to infection with $S$. Suipestifer among rats deprived of vitamin A, as compared with animals on a complete diet.

Dr. H. Chick and Miss Copping have found that vitamin $B_{2}$ is destroyed by strengths of alcohol above 50 per cent : its solubility and precipitation limits are uncertain and appear to depend on the compounds with which it may be associated in solution. Egg white is a convenient source of this vitamin since it contains no vitamin $\mathbf{B}_{1}$. Active concentrates have been prepared by aqueous acid extraction, which cure the dermatitis developing in rats on a vitamin $B_{2}$-free diet, but do not stimulate growth for more than a few weeks. Autoclaved yeast extracts are, however, satisfactory. It thus appears that a third factor is required by the rat for growth in addition to vitamins $B_{1}$ and $B_{2}$. This factor is also present in green-leaf vegetables, egg-yolk, and ox-liver, but absent from wheat embryo, meat, and white vegetables. Its thermostability

$$
\text { No. 3241, Vor. 128] }
$$


distinguishes it from the other $\mathrm{B}$ vitamins hitherto described. Miss Roscoe has found no relationship between fat in the diet and the need for vitamin $B_{2}$ : fat lessens the need for vitamin $B_{1}$.

Dr. W. R. Aykroyd has found that the endosperms of wheat, rice, and maize contain little vitamin $B_{1}$ as compared with the embyro or whole grain. Parboiled rice, however, even after milling, contains definite amounts, due apparently to diffusion inwards from the germ and pericarp during the boiling. Maize has been found a good source of vitamin $\mathrm{B}_{2}$, as good as wheat, and better than rice. Pellagra, however, occurs among maize-eating peoples and is rarely observed where rice is the staple food. This result is against the theory that pellagra is due to a simple deficiency of vitamin $\mathrm{B}_{2}$.

Miss E. M. Hume and Miss H. H. Smith have compared the effect on bone calcification in rats of diets low and rich in ergosterol, the animals being given a complete synthetic diet without vitamin $D$ but irradiated daily. The bone ash was slightly lower in the animals on the low ergosterol diet which were not irradiated than in any of the other groups. The diet used contained no fat : many of the rats developed scaliness of the tails, which has been attributed to absence of fat from the diet. Hume and Smith observed the condition, however, in other rats on a diet containing fat, so that it must be due to some other defect in the diet.

Dr. N. S. Lucas has reinvestigated the permeability of human epidermis to radiations between $4370 \mathrm{~A}$. and $2400 \mathrm{~A}$. The transparency has been much underestimated, since considerable scattering of the incident light occurs, so that it fails to reach the slit of the spectrograph, although it may ultimately be transmitted by the skin at a distance from its point of entry. When allowance is made for this, by producing scattering of the light before it reaches the skin, it is found that 55 per cent is transmitted at $3130 \mathrm{~A}$. and 26 per cent at $2940 \mathrm{~A}$. The absorption curve agrees closely with those of casein, serum albumin, tryptophane, and tyrosine.

Dr. Smedley-MacLean and Miss Pearce have shown that when a large excess of peroxide acts on oleic acid at $95^{\circ} \mathrm{C}$., less than 20 per cent of the carbon appears as formic, carbonic, and acetic acids, acids with 15 and 12 carbon atoms being obtained, that is, oxidation occurs first at the double bond followed by oxidation of the 18 carbon chain in the $\gamma$ position. In the presence of a cupric salt, the oxidation proceeds much further, 70 per cent appearing as the lower acids. At $60^{\circ} \mathrm{C}$. the chief products are 4-keto 7-hydroxy caprylic and succinic acids with 20 per cent of the lower acids. The evidence lends no support to the view that fatty acids are broken down by $\beta$-oxidation in the body. The presence of succinic acid is of interest since an oxidising enzyme for this acid is widely distributed throughout the tissues.

Among other researches with which the Report deals may be mentioned those of Dr. Robison on calcification in vitro and on the chemistry of the hexosephosphates and the work of Dr. V. Korenchevsky on the amount of fat in castrated and cryptorchid animals: degeneration or removal of the seminiferous tissue leads to an increase in fat deposition.

\section{A New Illumination Device for Microscopy.}

THE microscopic examination of opaque materials and the surface structure of natural objects have recently attracted much attention. The root idea of the Lieberkühn has been embodied in various types of ring illuminator in which the light may reach an annular reflector from below, or may be dealt with in a manner more analogous to the vertical illuminator.

Messrs. Ernst Leitz have recently brought out improved apparatus of the latter kind in which the light reaches the object from an annular illuminator around the objective, but avoiding the restriction in the size of the object which has been necessary in some arrangements. A small 8-volt lamp and condenser project a roughly parallel beam which is reflected downwards by an annular mirror held at $45^{\circ}$ to the beam and to the axis of the microscope tube. The reflector is pierced with a central aperture which transmits the upward image-forming rays from the objective. The annular beam thus produced from the reflector enters an annular lens (or reflector system for the higher powers) and is focused on the object without travers. ing the objective itself. Thus the disadvantages of back reflections and stray light are avoided. The general arrangement of the parts is shown in the accompanying illustration (Fig. 1).

A special series of fifteen new objectives, comprising 'dry', 'water immersion', and 'oil immersion ' lenses has been produced, in which the low-power lenses are designed to work without a cover glass over the object. Each of the low-power lenses has its own ring condenser, and rapid transition from one object to another is facilitated by the use of a convenient objective changer which clamps firmly and definitely into position. The lamp and condenser system need not be changed.

One advantage of apparatus of this kind is that the illumination can be controlled with much greater precision than with any more or less haphazard reflector arrangement, and that a systematic study of objects can therefore be commenced with less uncertainty. The interpretation of the images in some cases, especially with the higher powers, is not an easy matter,

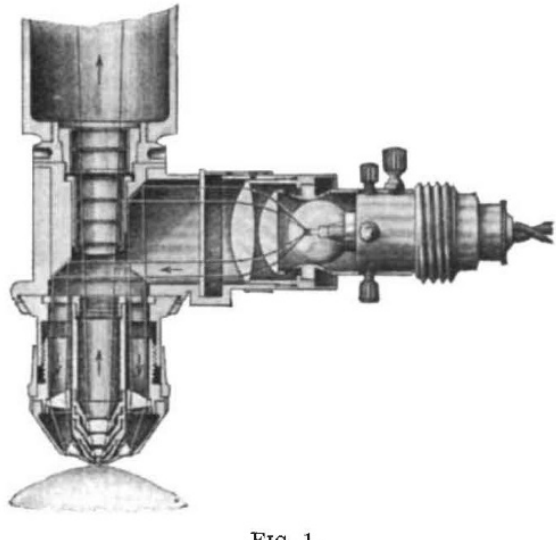

FIG, 1.

and will require careful study; diffraction effects can easily be mistaken for surface colour ; neverthe. less, it is clear that new investigations are possible. The well-illustrated catalogue of this 'Ultropaque Illuminator' gives photographs of various objects, amongst which are vegetable tissues, skin, chromatophores, leather, wood-sections, fabrics, and so on. This method may also have important possibilities in metallography. 